\title{
The Wiener Index and the Hosoya Polynomial of the Jahangir Graphs
}

\author{
Shaohui Wang ${ }^{1,2}$, Mohammad Reza Farahani, ${ }^{3,}$, M. R. Rajesh Kanna ${ }^{4}$, \\ Muhammad Kamran Jamil ${ }^{5}$, R. Pradeep Kumar \\ ${ }^{1}$ Department of Mathematics, University of Mississippi, University, MS, USA \\ ${ }^{2}$ Department of Mathematics and Computer Science, Adelphi University, Garden City, NY, USA \\ ${ }^{3}$ Department of Applied Mathematics, Iran University of Science and Technology (IUST) Narmak, Tehran, Iran \\ ${ }^{4}$ Department of Mathematics, Maharani's Science College for Women, Mysore, India \\ ${ }^{5}$ Department of Mathematics, Riphah Institute of Computing and Applied Sciences (RICAS), Riphah International University, Lahore, \\ Pakistan \\ ${ }^{6}$ Department of Mathematics, the National Institute of Engineering, Mysuru, India
}

\section{Email address:}

shaohuiwang@yahoo.com (S. Wang),mrfarahani88@gmail.com (M. R. Farahani),mr.rajeshkanna@gmail.com (M. R. R. Kanna), m.kamran.sms@gmail.com (M. K. Jamil),pradeepr.mysore@gmail.com (P. P. Kumar)

${ }^{*}$ Corresponding author

\section{To cite this article:}

Shaohui Wang, Mohammad Reza Farahani, M. R. Rajesh Kanna, Muhammad Kamran Jamil, R. Pradeep Kumar. The Wiener Index and the Hosoya Polynomial of the Jahangir Graphs. Applied and Computational Mathematics. Vol. 5, No. 3, 2016, pp. 138-141.

doi: $10.11648 /$ j.acm.20160503.17

Received: April 21, 2016; Accepted: May 3, 2016; Published: July 13, 2016

\begin{abstract}
Let $\mathrm{G}$ be a simple connected graph having vertex set $\mathrm{V}$ and edge set $\mathrm{E}$. The vertex-set and edge-set of $\mathrm{G}$ denoted by $V(G)$ and $E(G)$, respectively. The length of the smallest path between vertices $u, v \in V(G)$ is called the distance, $d(u, v)$, between the vertices u,v. Mathematical chemistry is the area of research engaged in new application of mathematics in chemistry. In mathematics chemistry, we have many topological indices for any molecular graph, that they are invariant on the graph automorphism. In this research paper, we computing the Wiener index and the Hosoya polynomial of the Jahangir graphs $\mathrm{J}_{5, \mathrm{~m}}$ for all integer number $\mathrm{m} \geq 3$. The Wiener index is the sum of distances between all pairs of vertices of $G$ as $W(G)=$ $\sum_{\{u, v\} \subset V(G)} d(u, v)$. And the Hosoya polynomial of $\mathrm{G}$ is $\mathrm{H}(\mathrm{G}, \mathrm{x})=\sum_{\{u, v\} \subset V(G)} x^{d(u, v)}$, where d(u,v) denotes the distance
\end{abstract} between vertices $u$ and $v$.

Keywords: Regular Graphs, Connected Graphs, Jahangir Graphs, Topological Indices, Hosoya Polynomial, Wiener Index, Distances

\section{Introduction}

Let $\mathrm{G}$ be a connected graph. The vertex-set and edge-set of $\mathrm{G}$ denoted by $V(G)$ and $E(G)$, respectively. The degree of a vertex $v \epsilon V(G)$ is the number of vertices joining to $v$ and denoted by $d_{v}$

Topological indices of a simple graph are numerical descriptors that are derived from graph of chemical compounds. Such indices based on the distances in graph are widely used for establishing relationships between the structure of molecular graphs and Nanotubes and their physicochemical properties. Usage of topological indices in biology and chemistry began in 1947 when chemist Harold Wiener [1] introduced the Wiener index to demonstrate correlations between physicochemical properties of organic compounds and the index of their molecular graphs. Wiener originally defined his index on trees and studied its use for correlations of physico-chemical properties of alkanes, alcohols, amines and their analogous compounds [2] as: 


$$
\mathrm{W}(\mathrm{G})=1 / 2 \sum_{v \in V(G)} \sum_{u \in V(G)} \mathrm{d}(\mathrm{u}, \mathrm{u})
$$

where $d(u, v)$ denotes the distance between vertices $u$ and $v$.

The Hosoya polynomial of a graph is a generating function about distance distributing, introduced by Haruo Hosoya in 1988 and for a connected graph $G$ is defined as [3]:

$$
\mathrm{H}(\mathrm{G}, \mathrm{x})=1 / 2 \sum_{v \in V(G)} \sum_{u \in V(G)} x^{d(v, u)}
$$

In a series of papers, the Wiener index and the Hosoya polynomial of some molecular graphs and Nanotubes are computed. For more details about the Wiener index and the Hosoya polynomial, please see the paper series [4-22] and the references therein.

In this research paper, we present some properties of the Wiener index and the Hosoya polynomial and we introduce a closed formula of this and the correspondent polynomial of the Jahangir graphs $J_{5, m}$ for all integer number $m \geq 3$.

\section{Materials and Methods}

The In the this section, we present some studies about a semi-regular and connected graphs that named "Jahangir Graphs $J_{n, m} " \forall n \geq 2, \forall m \geq 3$. And we introduce a method to compute the Wiener index and the Hosoya polynomial of the Jahangir graphs $J_{5, m}$ in continue.

What is a the Jahangir graphs $\mathrm{J}_{\mathrm{n}, \mathrm{m}}$ ?

Definition 2.1. [23, 24] The Jahangir graphs $\mathrm{J}_{\mathrm{n}, \mathrm{m}}$ is a graph on $n m+1$ vertices and $\mathrm{m}(\mathrm{n}+1)$ edges $\forall \mathrm{n} \geq 2 \quad \& \forall \mathrm{m} \geq 3$; i.e., a graph consisting of a cycle $\mathrm{C}_{\mathrm{nm}}$ with one additional vertex which is adjacent to $m$ vertices of $C_{n m}$ at distance $n$ to each other on $\mathrm{C}_{\mathrm{nm}}$.

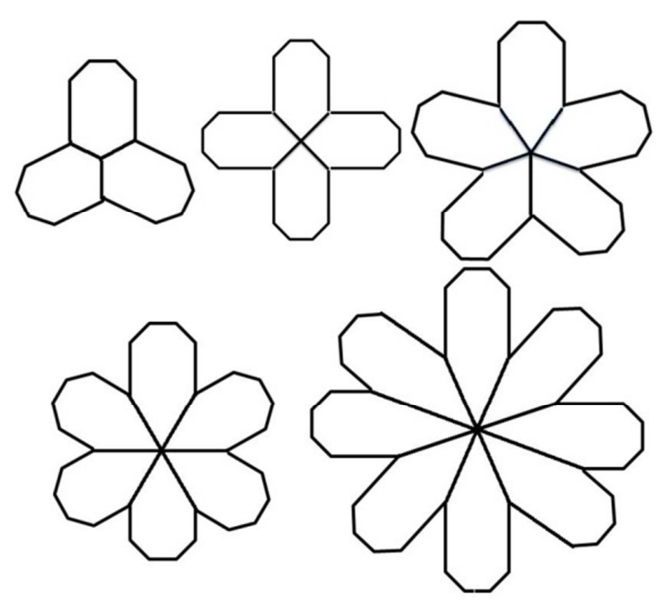

Figure 1. Some examples of Jahangir graphs $J_{5,3}, J_{5,4}, J_{5,5}, J_{5,6}$ and $J_{5,8}$.

In particular, $\forall \mathrm{m} \geq 3$ the Jahangir graphs $\mathrm{J}_{5, \mathrm{~m}}$ is a graph consisting of a cycle $\mathrm{C}_{5 \mathrm{~m}}$ with one additional vertex (the Center vertex c) which is adjacent to $m$ vertices of $\mathrm{C}_{\mathrm{nm}}$ at distance 5 to each other on $\mathrm{C}_{5 \mathrm{~m}}$. Some first example of this graphs are shown in Figure 1. For more details about the Jahangir graphs $\mathrm{J}_{\mathrm{n}, \mathrm{m}}$ reader can see the paper series [23-33, 34-36].

\section{Main Results and Discussion}

In this paper, we computed a closed formula of the Wiener index and the Hosoya polynomial of the Jahangir graphs $J_{5, m}$ for all integer number $m \geq 3$.

Theorem 2.1. The Hosoya polynomial and the Wiener index for the Jahangir graphs $J_{5, m}$ for all integer number $m \geq 3$ are equal to

$$
\begin{gathered}
H\left(J_{5, m}, x\right)=6 m x^{1}+1 / 2\left(m^{2}+13 m\right) x^{2}+\left(2 m^{2}+5 m\right) x^{3}+\left(4 m^{2}-4 m\right) x^{4} \\
+\left(4 m^{2}-6 m\right) x^{5}+\left(2 m^{2}-5 m\right) x^{6} \\
W\left(J_{5, m}\right)=55 m^{2}-42 m .
\end{gathered}
$$

Proof. Let $J_{5, m}$ be Jahangir graphs $\forall m \geq 3$ with $5 m+1$ vertices and $6 m$ edges. From Definition 2.1 and Figure 1, one can see that there are $4 m$ vertices of the Jahangir graph $J_{5, m}$ with degree 2 and $m$ vertices of $J_{5, m}$ with degree 3 and Center vertex $c$ has degree $m$ and we have three partitions of the vertex set $V\left(J_{5, m}\right)$ as follow

$$
\begin{aligned}
& V_{2}=\left\{v \in V\left(J_{5, m}\right) \mid d_{v}=2\right\} \rightarrow\left|V_{2}\right|=4 m \\
& V_{3}=\left\{v \in V\left(J_{5, m}\right) \mid d_{v}=3\right\} \rightarrow\left|V_{3}\right|=m \\
& V_{m}=\left\{c \in V\left(J_{5, m}\right) \mid d_{c}=m\right\} \rightarrow\left|V_{m}\right|=1
\end{aligned}
$$

And alternatively, $V\left(J_{5, m}\right)=V_{2} \cdot \cup V_{3} \cup V_{m}$ and $V_{2} \cdot \cap V_{3} \cap V_{m}=\varnothing$ and we know

$$
|\mathrm{E}(\mathrm{G})|=1 / 2 \sum_{k=\delta}^{\Delta}\left|V_{k}\right| \times k
$$

where $\delta$ and $\Delta$ are the minimum and maximum of $d_{v}$ for all $v \in V(G)$, respectively, thus

$$
\left|\mathrm{E}\left(\mathrm{J}_{5, \mathrm{~m}}\right)\right|=1 / 2\left[2 \times\left|\mathrm{V}_{2}\right|+3 \times\left|\mathrm{V}_{3}\right|+3 \times\left|\mathrm{V}_{\mathrm{m}}\right|\right] .
$$

Now, for compute the Hosoya polynomial and the Wiener index of $J_{5, m}$, we denote the number of unordered pairs of vertices $u$ and $v$ of a graph $G$ as distance $d(\mathrm{u}, \mathrm{v})=k$ by $d(G, k)$ for all integer number $k$ up to $d(G)$ (where $\mathrm{d}(G)$ denote the topological diameter and is the longest distance between vertices of a graph $G$ ). Thus, we redefine this mention topological polynomial and index of $G$ as follow:

$$
\begin{aligned}
& H(G, x)=\sum_{k=1}^{d(G)} d(G, k) x^{k} \\
& W(G)=\sum_{k=1}^{d(G)} d(G, k) \times k
\end{aligned}
$$

From the Definition 2.1 and the structure of Jahangir graphs $J_{5, m}$ in Figure 1, we have following computations for $d\left(J_{5, m}, k\right)\left(\forall k: 1 \leq k \leq d\left(J_{5, m}\right)\right)$ :

$$
\begin{gathered}
d\left(J_{5, m}, 1\right)=\left|E\left(J_{5, m}\right)\right|=6 m \text { by definitions of } d(G, k) . \\
d\left(J_{5, m}, 2\right)=1 / 2 m(m+13)
\end{gathered}
$$

Since, there are two 2-edges paths between Center vertex $c \epsilon V\left(J_{5, m}\right)$ and other vertices of vertex set $\left.V_{2} \subset V\left(J_{5, m}\right)\right)$. $1 / 2\left|V_{3}\right|(m-1)$ 2-edges paths between all vertices of $u, v \epsilon V_{3} \subset V\left(J_{5, m}\right)$ ), and $2\left|V_{3}\right|+\left|V_{3}\right|$ 2-edges paths start from vertices of $V_{2}$ until vertices of $V_{3}$ and $V_{2} \subset V\left(J_{5, m}\right)$. Thus the 
second sentence of the Hosoya polynomial $H\left(J_{5, m}, x\right)$ of $J_{5, m}$ is equal to $\left[2\left|V_{3}\right|+1 / 2\left|V_{3}\right|(m-1)+3\left|V_{3}\right|+2\left|V_{3}\right|\right] x^{2}=1 / 2\left(m^{2}+13 m\right) x^{2}$.

$$
d\left(J_{5, m}, 3\right)=\left(2 m^{2}+5 m\right)
$$

Because, there are $2\left|V_{3}\right|$ 3-edges paths between $c \epsilon V_{m}$ and vertices of $V_{2}$, but there are not any 3-edges paths between $c$ and vertices of $V_{3}$. Also, there are $1 / 2 \times 2\left|V_{3}\right|+2\left|V_{3}\right|=3 m 3$ edges paths between all vertices of $\left.u, v \in V_{2} \subset V\left(J_{5, m}\right)\right)$. From Figure 1, one can see that $2\left|V_{3}\right|+\left|V_{3}\right|(2(m-1))$ 3-edges paths started from vertices of $V_{3}$ until vertices of $V_{2} \subset V\left(J_{5, m}\right)$. Thus, the third sentence of $H\left(J_{5, m}, x\right)$ is $1 / 2$ $\left[2\left|V_{3}\right|+0+3\left|V_{3}\right|+2 \mathrm{~m}\left|V_{3}\right|\right] x^{3}=m(2 m+5) x^{3}$.

$$
d\left(J_{5, m}, 4\right)=4 m^{2}-2 m
$$

Since from Figure 1, there are $2\left|V_{3}\right|$ 3-edges paths between $c \epsilon V_{m}$ and vertices of $V_{2}$. And obviously there are not any 4edges paths between vertices of $V_{3}$ and $c$. And there are $\left|V_{3}\right|\left(2\left|V_{3}\right|-4\right)$ 4-edges paths between vertices of $V_{3}$ and $V_{2}$. Also, there are $d\left(C_{m}\right)+1 / 2 \times 2\left|V_{3}\right|\left(2\left|V_{3}\right|-3\right)$ 4-edges paths between all vertices of $\left.u, v \epsilon V_{2} \subset V\left(J_{5, m}\right)\right)$. Therefore the coefficient of the fourth sentence of $H\left(J_{5, m}, x\right)$ is $2\left|V_{3}\right|+0+\left|V_{3}\right|\left(2\left|V_{3}\right|-4\right)+2 m(m-1)=4 m(m-1)=4 m^{2}-4 m$.

$$
d\left(J_{5, m}, 5\right)=\left(4 m^{2}-6 m\right)
$$

From the Definition 1 and the structure of $J_{5, m}$, one can see that there are not any 5-edges paths between vertices of $V_{3}$ and $c$ and vertices of $V_{2}$ and between vertices of $u \epsilon V_{2}$ and $v \in V_{3}$. Thus for the $5^{\text {th }}$ sentence of the Hosoya polynomial of $J_{5, m}$, we have $2 d\left(C_{m}\right)+1 / 2 \times\left|V_{2}\right|\left(2\left|V_{3}\right|-4\right)=2 m(2 m-3) \quad 5$-edges paths between all vertices of $u, v \in V_{2}$.

$$
\text { For } \mathrm{d}\left(\mathrm{J}_{5, \mathrm{~m}}\right)=6 ; \mathrm{d}\left(\mathrm{J}_{5, \mathrm{~m}}, 6\right)=\left(2 \mathrm{~m}^{2}-5 \mathrm{~m}\right)
$$

Because, there isn't any 6-edges paths started from members of $C$ and $V_{3}$ to all other vertices of Jahangir graphs $J_{5, m}$ in Figure 1. But there are $1 / 2 \times\left|V_{2}\right|\left(2\left|V_{2}\right|-5\right)=m(2 m-5) 6$ edges paths between all vertices $V_{2}$. Thus, the 6 th and last sentence of $H\left(J_{5, m}, x\right)$ is $m(2 m-5) x^{6}$.

Now, Equations 12, 13,.., 17 imply that the Hosoya polynomial of the Jahangir graph $J_{5, m}$ is equal to:

$$
\begin{aligned}
H\left(J_{5, m}, x\right) & =6 m x^{1}+1 / 2\left(m^{2}+13 m\right) x^{2}+\left(2 m^{2}+5 m\right) x^{3} \\
& +\left(4 m^{2}-4 m\right) x^{4}+\left(4 m^{2}-6 m\right) x^{5}+\left(2 m^{2}-5 m\right) x^{6}
\end{aligned}
$$

Also, from the definition of Wiener index and the Hosoya Polynomial of a graph $G$ and the first derivative of Hosoya polynomial (evaluated at $x=1$ in Equations 10,11), we cam compute the Wiener index of the Jahangir graph $J_{5, m}$ as follow:

$$
\begin{gathered}
W\left(J_{5, m}\right)=\left.H\left(J_{5, m}, x\right){ }^{\prime}\right|_{x=1} \\
=\sum_{k=1}^{d\left(J_{5, m}\right)} d\left(J_{5, m}, k\right) \times k \\
=\left[6 m x^{1}+1 / 2\left(m^{2}+13 m\right) x^{2}+\left(2 m^{2}+5 m\right) x^{3}+\left(4 m^{2}-4 m\right) x^{4}+\left(4 m^{2}-\right.\right. \\
\left.6 m) x^{5}+\left(2 m^{2}-5 m\right) x^{6}\right]\left.\right|_{x=1} \\
=[6 m \times 1+1 / 2 m(m+13) \times 2+m(2 m+5) \times 3+4 m(m-1) \times 4+m(4 m- \\
6) \times 5+m(2 m-5) \times 6] \\
=55 m^{2}-42 m .
\end{gathered}
$$

Here the proof of theorem is completed.

Example 2.1. The Hosoya polynomial and the Wiener index for $J_{5,6}$ are equal to:

$$
\begin{gathered}
H\left(J_{5,6}, x\right)=36 \mathrm{x}+57 \mathrm{x}^{2}+102 \mathrm{x}^{3}+120 \mathrm{x}^{4+} 108 \mathrm{x}^{5}+42 \mathrm{x}^{6} \\
W\left(J_{5,6}\right)=55(6)^{2}-42(6)=1728
\end{gathered}
$$

\section{Conclusion}

In this paper, we obtained the Hosoya polynomial and the Wiener index of graph "the Jahangir graphs $J_{5, m}$ for all integer number $m \geq 3$ " for the first time as:

$$
\begin{gathered}
H\left(J_{5, m}, x\right)=6 m x^{1}+1 / 2\left(m^{2}+13 m\right) x^{2}+\left(2 m^{2}+5 m\right) x^{3}+\left(4 m^{2}-4 m\right) x^{4} \\
+\left(4 m^{2}-6 m\right) x^{5}+\left(2 m^{2}-5 m\right) x^{6} \\
W\left(J_{5, m}\right)=55 m^{2}-42 m .
\end{gathered}
$$

\section{Acknowledgements}

The author is thankful to Professor Emeric Deutsch from Department of Mathematics of Polytechnic University (Brooklyn, NY 11201, USA) for his precious support and suggestions.

\section{References}

[1] H. Wiener, Structural determination of paraffin boiling points, J. Amer. Chem. Soc. 69 (1947), 17-20.

[2] P. V. Khadikar and S. Karmarkar. On the estimation of PI index of polyacenes. Acta Chim. Slov. 49, 2002, 755-771.

[3] H. Hosoya. On some counting polynomials in chemistry. Discrete Appl. Math. 19, (1988), 239-257.

[4] Gutman, O. E. Polansky, Mathematical Concepts in Organic Chemistry Springer, Berlin, 1986.

[5] Gutman, S. Klavžar, B. Mohar. Fifty years of the Wiener index, (Eds.), MATCH Commun. Math. Comput. Chem. 35, $1-259$ (1997).

[6] Gutman, S. Klavžar, B. Mohar. Fiftieth anniversary of the Wiener index, (Eds.), Discrete Appl. Math. 80, 1--113 (1997).

[7] E. Estrada, O. Ivanciuc, I. Gutman, A. Gutierrez and L. Rodrguez, Extended Wiener Indices. A New Set of Descriptors for Quantitative Structure Property Studies, New J. Chem. 22 (1998) 819-823.

[8] H. Hosoya, A newly proposed quantity characterizing the topological nature of structural isomers of saturated hydrocarbons, Bull. Chem. Soc. Japan 44 (1971), 2332-2339.

[9] Gutman, S. Klavžar, M. Petkovsek and P. Zigert, On Hosoya polynomials of benzenoid graphs, MATCH Commun. Math. Comput. Chem., 43 (2001) 49-66.

[10] A. Dobrynin, R. Entringer, I. Gutman, Wiener index of trees: Theory and applications, Acta Appl. Math. 66 (2001), 211-249.

[11] M. V. Diudea. Hosoya polynomial in Tori. MATCH Commun. Math. Comput. Chem. 45, (2002), 109-122. 
[12] M. Eliasi, B. Taeri, Hosoya polynomial of zigzag polyhex Nanotorus, J. Serb. Chem. Soc. 73 (2008) 313-319.

[13] S. Xu, H. Zhang, Hosoya polynomials of armchair openended Nanotubes, Int. J. Quant. Chem. 107 (2007) 586-596.

[14] J. Chen, S. Xu, H. Zhang, Hosoya polynomials of $\mathrm{TUC}_{4} \mathrm{C}_{8}(\mathrm{R})$ Nanotubes, Int. J. Quant. Chem. 109 (2009) 641-649.

[15] S. Xu, H. Zhang, The Hosoya polynomial decomposition for catacondensed benzenoid graphs, Discr. Appl. Math. 156 (2008) 2930-2938.

[16] H. Shabani, A. R. Ashrafi, Applications of the matrix package MATLAB in computing the Wiener polynomial of armchair polyhex nanotubes and nanotori, J. Comput. Theor. Nanosci. 7 (2010) 1143-1146.

[17] M. Knor, P. Potočnik and R. Škrekovski. Wiener index of iterated line graphs of trees homeomorphic to the claw $\mathrm{K}_{1 ; 3}$. Ars Math. Contemp. 6 (2013), 211-219.

[18] M. R. Farahani. Hosoya polynomial, Wiener and HyperWiener indices of some regular graphs. Informatics Engineering, an International Journal (IEIJ), 1 (1), (2013), 913.

[19] M. R. Farahani, M. P. Vlad. On the Schultz, Modified Schultz and the Hosoya polynomials and Derived Indices of Capradesigned planar Benzenoid. Studia UBB Chemia. 57 (4), (2012), 55-63.

[20] M. R. Farahani. Schultz indices and Schultz Polynomials of Harary graph. Pacific Journal of Applied Mathematics. 6 (3), (2014), 77-84.

[21] M. R. Farahani. Hosoya, Schultz, Modified Schultz Polynomials and Their Topological Indices of Benzene Molecules: First Members of Polycyclic Aromatic Hydrocarbons (PAHs). International Journal of Theoretical Chemistry. 1 (2), (2013), 09-16.

[22] M. R. Farahani. On the Schultz polynomial, Modified Schultz polynomial, Hosoya polynomial and Wiener index of Circumcoronene series of benzenoid, J. Applied Math. \& Info. 31 (5-6), (2013). 595-608.

[23] K. Ali, E. T. Baskoro and I. Tomescu, On the Ramzey number of Paths and Jahangir graph $J_{3, m}$. 3rd International
Conference on 21st Century Mathematics 2007, GC University Lahoor Pakistan, March 2007.

[24] D. A. Mojdeh and A. N. Ghameshlou. Domination in Jahangir Graph $J_{2, m}$. Int. J. Contemp. Math. Sciences, 2 (24), (2007), 1193-1199.

[25] Lourdusamy. Covering Cover Pebbling Number for Jahangir Graph $J_{2, m}$. Sciencia Acta Xaveriana. 4 (2), 2012, 59-64.

[26] Lourdusamy, S. Samuel Jeyaseelan and T. Mathivanan. On Pebbling Jahangir Graph. Gen. Math. Notes, 5 (2), 2011, 4249.

[27] Lourdusamy and T. Mathivanan. The 2t-Pebbling Property on the Jahangir Graph $J_{2 ; m}$. Gen. Math. Notes, 28 (1), 2015, 1839.

[28] Lourdusamy and T. Mathivanan. The t-pebbling number of Jahangir graph $J_{3, m}$. Proyecciones Journal of Mathematics. 34 (2), 161-174, 2015

[29] Lourdusamy, S. Samuel Jeyaseelan and T. Mathivanan. The tPebbling Number of Jahangir Graph. International J. Math. Combin. 1, (2012), 92-95.

[30] M. Ramachandran and N. Parvathi. The Medium Domination Number of a Jahangir Graph $J_{m, n}$. Indian Journal of Science and Technology, 8 (5), 400-406, 2015

[31] M. R. Farahani, Hosoya Polynomial and Wiener Index of Jahangir graphs $J_{2, m}$. Pacific Journal of Applied Mathematics. 7 (3), 2015, In press.

[32] M. R. Farahani, The Wiener Index and Hosoya polynomial of a class of Jahangir graphs $J_{3, m}$. Fundamental Journal of Mathematics and Mathematical Science. 3 (1), 2015, 91-96.

[33] M. R. Farahani, Hosoya Polynomial of Jahangir graphs $J_{4, m}$. Global Journal of Mathematics, 3 (1), 2015, 232-236.

[34] S. Wang, B. Wei, Multiplicative Zagreb indices of Cactus graphs, Discrete Mathematics, Algorithms and Applications, DOI: $10.1142 / \mathrm{S} 1793830916500403$.

[35] C. Wang, S. Wang, B. Wei, Cacti with Extremal PI Index, Transactions on Combinatorics, Vol. 5 No. 4 (2016), pp. 1-8.

[36] S. Wang, B. Wei, Multiplicative Zagreb indices of k-trees, Discrete Applied Mathematics, 180 (2015), 168-175. 